\title{
An integrated agent-based model of travel demand and package deliveries
}

\author{
Anna Reiffer ${ }^{a *}$, Jelle Kübler $^{a}$, Lars Briem $^{a}$, Martin Kagerbauer ${ }^{a}$, Peter Vortisch $^{a}$
}

${ }^{a}$ Institute for Transport Studies, Karlsruhe Institute of Technology (KIT), Kaiserstr. 12, 76131 Karlsruhe, Germany

\begin{abstract}
With the increased shift from in-store shopping to e-commerce, we can expect the share of delivery vehicles in cities to rise as well. This puts great pressure on cities and surrounding areas as emissions rise and space becomes scarce. Because the last leg of the delivery chain is so costly, there have been many studies on how the last-mile issue can be diminished. However, most studies only cover deliveries independently from the orders. This paper presents a modelling approach that integrates travel demand, package orders and subsequent deliveries within the same framework. One of the benefits of the integrated modelling approach is that the model can evaluate the agents' locations at all times during the simulation. This allows us to model different states of delivery on a microscopic level. We have applied the model to Karlsruhe, Germany with a synthetic population of just over 300.000 agents. The results show that the model simulates both package orders and the subsequent delivery tours realistically. The model framework allows for detailed analysis of delivery states and success rates and can be used for scenario analysis of different delivery methods and changes in online shopping behaviour.
\end{abstract}

Keywords: last-mile, agent-based, travel demand, urban freight

\section{Introduction}

Urban goods movements have increased rapidly in recent years especially due to the rising popularity of e-commerce. In 2019, the revenue of courier, express, and parcel (CEP) services in Germany amounted to $€ 21,3$ mio., twice as much as it was in 2000 [1]. With this increase, we can expect the share of delivery vehicles in cities to rise as well. This puts great pressure on cities and surrounding areas as emissions rise and space becomes scarce. Because the last leg of the delivery chain is so costly, there have been many studies on how the last-mile issue can be diminished.

Electric vehicles, for example, can replace conventionally powered delivery vehicles rather easily as usability remains the same. They can compensate emissions and have been put into practice already. However, they are still not an encompassing solution to the problems of last-mile delivery [2] and further solutions have to be investigated. One of such are parcel lockers which are used to drop off and pick up packages. They can be used by both the delivery service and the customer.
When the number of lockers and their locations are optimised they have the potential to especially reduce the number of failed deliveries [3]. In an effort to ban large delivery vehicles from cities altogether, (case) studies have also investigated the effect and efficiency of cargo-bicycles [4], [5].

Policy makers and CEP service providers are interested in understanding the effects of different last-mile delivery solutions and how they influence travel behaviour of the customer. Demand models and simulations are an established tool in assessing policy changes and effects on the transport system. However, existing models of urban freight movements have been limited to the delivery vehicles and in some cases their effect on the transport system. To assess effects of different delivery solutions not only on the transportation system but also on the customers' travel behaviour, we have integrated last-mile deliveries into the agent-based travel demand modelling framework mobiTopp.

Research on freight modelling has been quite extensive and with increased computational power, agent-based models are used more and more due to their analytical advantage over aggregate models. Agent-based models allow for consideration of different stakeholders in one model. One such model is the MASS-GT model presented by de Bok and Tavasszy, and de Bok et al. [6], [7] respectively. The authors avail the agent-

${ }^{*}$ Corresponding author.

Tel.: +49 608 47735, E-mail: anna.reiffer@ kit.edu

(C) 2021 International Association for Sharing Knowledge and Sustainability.

DOI: $10.5383 /$ JTTM.03.02.003 
based approach to differentiate between producers, receivers/consumers, shippers, carriers, own account carriers and, third party logistics which allows them to use the model as an assessment tool for logistics policy strategies. Joubert et al. [8] used the existing agent-based simulation framework MATSim to simulate both commercial and private vehicles. Due to the underlying data, the model does not differentiate between different trip purposes and thus delivery vehicles are not explicitly modelled. However, the authors' approach highlights the importance of modelling both private and commercial vehicles in the same model. This also holds true for the work of Schröder and Liedtke [9]. They designed a sandbox MATSim model in which both passenger and freight agents were regarded. The authors showed that policy measures aimed at freight transport may also have an effect on the utility of private transport. Another agent-based approach was presented by Alho et al. and Sakai et al., respectively [10], [11]. The authors used the modelling framework SimMobility and integrated urban freight movements. SimMobility Freight allows for analysis of operational efficiency. In a case study, Sakai et al. present the effects of nighttime and off-peak deliveries.

These models all utilise the advantages of agent-based models to analyse effects of freight policy and relationships between different stakeholders, however, they do not take the relationship between online shopping and travel behaviour into account. This connection, however, has been widely covered in previous research. Mokhtarian [12] for example investigated the impacts of business to customer (b2c) e-commerce on transportation and found that e-commerce can both reduce and increase personal travel depending on the individual circumstances. Weltevreden and Rotem-Mindali presented the effects of b2c and customer to customer (c2c) e-commerce in the Netherlands. The authors showed that b2c e-commerce reduces personal travel while $\mathrm{c} 2 \mathrm{c}$ e-commerce has the opposite effect. While there is an extensive body of research on this relationship (for a comprehensive literature review, see Bönisch et al. [13]), it has yet to be regarded in travel demand models. With this study, we aim to close this gap by incorporating the online shopping behaviour and subsequent travel behaviour into the agent-based travel demand modelling framework mobiTopp. We translate the orders into packages whose deliveries are simulated simultaneously with all other trips in the travel demand model.

This paper presents the modelling framework developed to account for last-mile package delivery in an agent-based travel demand model. After presenting mobiTopp, we detail the package delivery module logiTopp and the data used for the choice models. We go on to describe the results of the simulation, which we discuss in the subsequent section. We conclude this paper by addressing main outcomes of our work and implications for future work.

\section{Modelling Framework}

\section{1. mobiTopp}

We used the agent-based travel demand modelling framework mobiTopp [14], [15] to integrate last-mile package deliveries. As illustrated on the left-hand side of

Figure 1 mobiTopp is made up of two parts: the long-term and the short-term module. In the long-term module, a synthetic population is generated consisting of individual people in the model area and their corresponding households. The generated people are the agents in the model and they each have the following characteristics attributed to them: age, gender, work status, place of work/education, drivers license, commuter tickets, and memberships to mobility services (e.g. carsharing, bikesharing, etc.). The attributes differentiated for each household are the number of household members, the number of cars, the location of the household, and its net income. Based on the personal and respective household characteristics, each agent is assigned an activity schedule for the simulation period which can be set to up to a week [16]. In mobiTopp, we differentiate six main activities: work, education, shopping, leisure, service, and home. These can be further refined to allow for a more differentiated model. Within the long-term module, each activity is assigned a preliminary start time and duration.

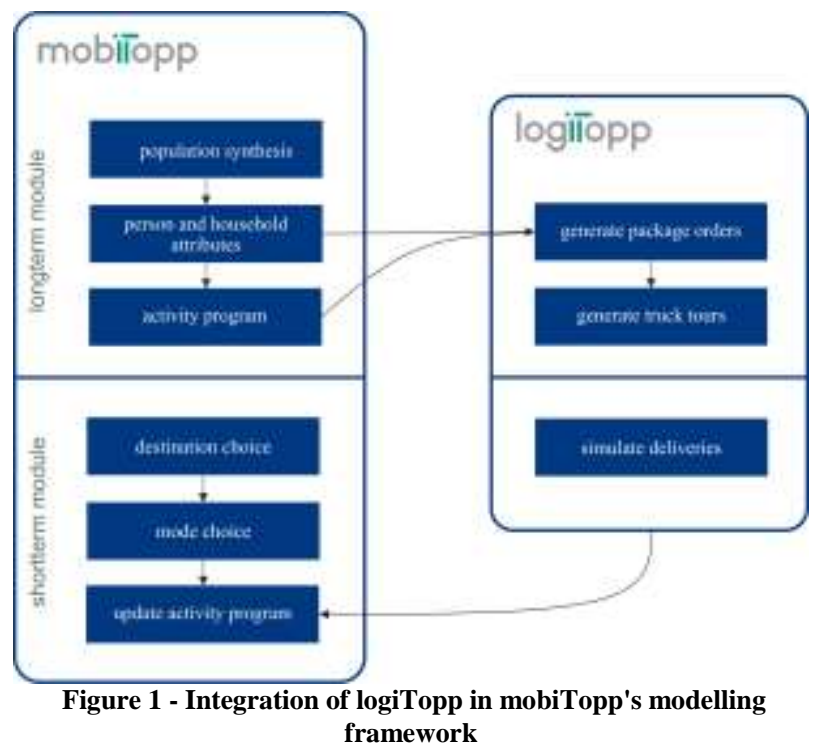

These activities are then simulated in the short-term module of the modelling framework. Within this module, a destination and a mode is chosen for the next activity. This process is repeated each time an activity is finished. As the activity schedules created in the long-term module work with estimated travel times between activities, they need to be updated according to the actual travel times which is also part of the simulation process. The simulation runs simultaneously for all agents which allows for a realistic interaction between agents in mobiTopp. 


\section{2. logiTopp}

We have used the interaction between agents and the high level of detail regarding their characteristics to integrate last-mile package deliveries in an urban area. The model extension logiTopp uses information from mobiTopp's long-term module to generate package orders in the study area. The points of integration are illustrated in

Figure 1. We use the characteristics of the agents and their households created in the population synthesis and activity scheduling part of mobiTopp to model package order behaviour. The packages are generated with their delivery location and distributed among delivery centres in the model area. For each delivery agent of the distribution centres, a delivery tour is formed and simulated. Depending on the delivery location, the activity schedule of the receiving agents is updated such that they include the pick up of their packages. Like mobiTopp, logiTopp is implemented in Java and available as an open source project on GitHub [17].

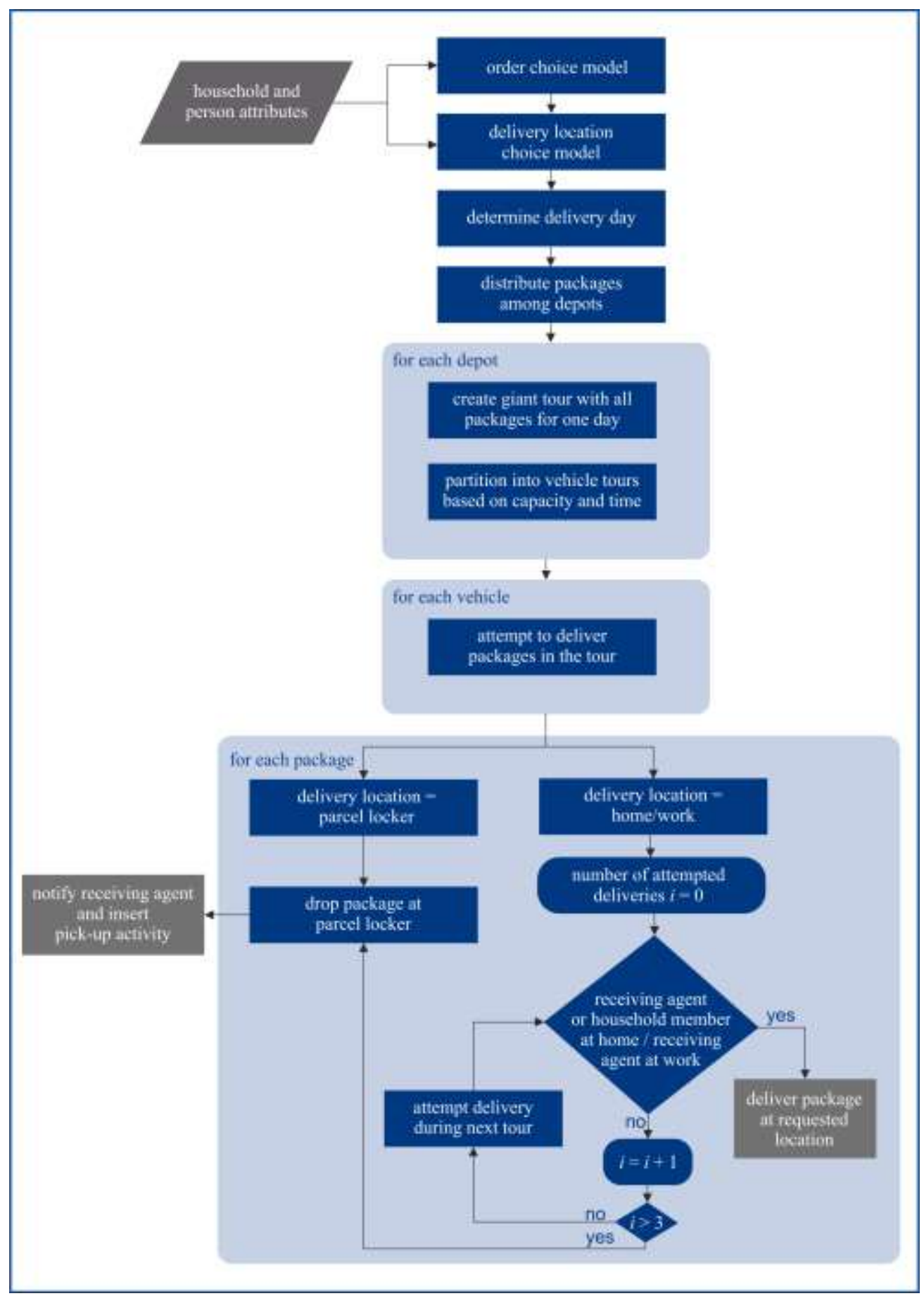

Figure 2 - Detailed flowchart of logiTopp 
We estimate the package orders using a multinomial logit model in which the number of packages ordered by each agent is the dependent variable and the attributes and characteristics from the long-term module, i.e. person and household attributes and activity patterns serve as the independent variables. We have defined the utility of agent $i$ ordering $n$ packages as:

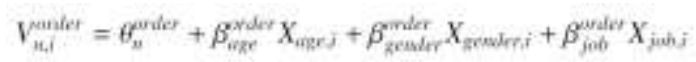

where $\theta$ is the alternative specific constant, $X$ is the respective attribute of agent $i$ and $\beta$ are the model parameters.

After the number of package orders per agent is determined, a respective place of delivery is chosen. We differentiate the alternatives home, work and parcel locker. The utility function of agent $i$ choosing delivery location $m$ is currently defined using the same information as in the order choice model:

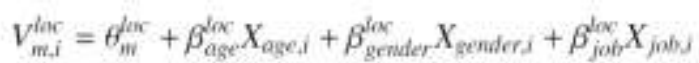

We used Monte Carlo simulations to determine the number of packages and their locations respectively based on their utilities. We recognise that the choice models are still rudimentary and are currently used as a technical prototype. Both choice models will be improved upon in future work to account for more factors influencing online shopping behaviour for example household characteristics and income. The package orders are simulated for the entire simulation period.

Once the packages and their respective delivery location is determined, they are allocated to package distribution centres. We use the actual distribution centres located in the mode area with their location. There is only one CEP service provider that operates two distribution centres for our example region, all other service providers only operate one centre. Thus, the distribution among the centres is only determined by the size of the distribution centres and no other factors were considered.

After the packages are allocated to the distribution centres, the tours of the delivery trucks are formed. Finding the optimal sequence of deliveries for each vehicle is an application of the Vehicle Routing Problem (VRP). Because the VRP is NP-hard, solutions are often approximated using heuristics. One such heuristic is the route first cluster second approach [18]. In this approach, the first step is to build a giant tour where the constraint of vehicle capacity is relaxed and a route for all packages within one tour is determined. In the next step, the giant tour is partitioned such that conditions of vehicle capacities are met. Generating the giant tour can also be defined as the travelling salesman problem (TSP) which is also NP-hard. To form the giant tour for each distribution centre we used the Java library JGraphT [19] that approximates a solution to the TSP. To form the tour for each vehicle we partitioned the giant tour using two constraints: time and vehicle capacity. With an approximated time to deliver a package and time between stops, we constrained the maximum duration of the tour including the trip back to the distribution centre to 8 hours, i.e. one working day. Each delivery agent conducts one tour per day. As we have currently not implemented a model for shipment size, the vehicle capacity is normal distributed around a mean value of 160 packages with a variance of 16 packages. The min. and max. values of packages are 100 and 200, respectively. The delivery agents are regular mobiTopp agents who have had a distribution centre assigned as their workplace as part of mobiTopp's longterm module in the population synthesis. Their activity program is now updated such that the delivery activities are integrated.

After the tour formation step, the simulation is run and the delivery agents start to deliver the packages of their tour. For each package, the model checks the chosen delivery location. Depending on the delivery location different conditions have to be met for the delivery agent to be able to successfully deliver the package:

- Delivery location $=$ work: When a package was ordered to a place of work, the delivery agent can deliver the package if the agent who ordered the package is at work during the time of delivery. This approach allows for a realistic delivery behaviour as most packages cannot be delivered to a place of work on Saturdays and delivery attempts would be unsuccessful.

- Delivery location = parcel locker: If the delivery agent has to deliver packages to a parcel locker the delivery is always successful. At this state of the model, we assume that there is always enough space left for the packages on the current tour. The parcel lockers for each agent are currently based in their home zone.

- Delivery location $=$ home: A delivery to the home of an agent works similarly to the delivery to a place of work The model checks if the agent who ordered the package or any other household member is currently at home. If that is the case the package is delivered successfully. The agent-based approach and simultaneous simulation of activities also allows for the model to check how many neighbours are at home at any given time during the simulation.

The strategy for packages that could not be delivered is currently the same for all delivery service providers: Whenever a delivery agent fails to deliver a package they take it back to the depot at the end of the tour and try again on their next tour, i.e. next day. This process is repeated three times. After the last delivery attempt, the delivery agent drops the package at a parcel locker. This delivery strategy and the analysis of different strategies require a multi-day simulation approach, which we are able to apply using mobiTopp. In the case that a package is delivered to a parcel locker after several failed delivery attempts, the agent who ordered the package is notified that their package is now in a parcel locker and ready for pick up. The activity program is then updated and the pickup activity is inserted into the program. The tours are reformed after each simulation day to account for packages that could not be delivered on the previous day and need to be considered in during the next tour.

\section{Results and Discussion}

We have chosen Karlsruhe, Germany as a model area for the first application of logiTopp. Karlsruhe is a city in the South-West of Germany and populated with just over 300,000 people. The area is serviced by nine parcel delivery companies which are represented in the model with their geo-locations. mobiTopp's synthetic population of Karlsruhe and it's surrounding areas includes about 1.9 mio. agents in about $879 \mathrm{k}$ households. We limited orders to agents living in the city of Karlsruhe as the shorter runtime allows us to analyse results faster and because most policies regarding last-mile delivery 
traffic target cities. The synthetic population of the regarded model area includes 303.809 agents in 170.013 households. Based on this population, logiTopp generates 136,399 packages for the simulation period of one week that are distributed among the delivery centres.

There are currently no survey data available for Karlsruhe which we could use for calibration. However, the German federal association of parcel and express logistics (BIEK) regularly conducts surveys of courier, express and parcel services. Based on the data published for the cities of Berlin, Hamburg and Munich [20] we extrapolated respective data for Karlsruhe. Table 1 shows the data from Berlin, Hamburg and Munich, the extrapolated data for Karlsruhe and the corresponding simulation results. This shows that our model simulates fewer packages than expected. This, however, can be attributed to the fact that we currently only model package deliveries to private entities, i.e. b2c and c2c deliveries. The two segments accounted for $70 \%$ of German package deliveries in 2019 [20]. Considering this proportion of sales segments, our model simulates private package deliveries realistically.

Table 1 - Daily and weekly package deliveries in German cities and model area

\begin{tabular}{lrrr}
\hline city & population & $\begin{array}{r}\text { packages } \\
\text { p. day }\end{array}$ & $\begin{array}{r}\text { packages p. week } \\
\text { (packages p. d. x 6) }\end{array}$ \\
\hline Berlin $^{1}$ & $3,669,491$ & 376,800 & $2,260,800$ \\
Hamburg $^{1}$ & $1,847,253$ & 208,300 & $1,249,800$ \\
Munich $^{1}$ & $1,484,226$ & 160,700 & 964,200 \\
Karlsruhe $^{2}$ & \multirow{2}{*}{303,809} & 32,783 & 196,697 \\
Simulation & & 22,733 & 136,399 \\
\hline 1- Source: [20]; 2 - extrapolated data & &
\end{tabular}

Packages can be delivered between Monday and Saturday from 8 a.m. to 8 p.m., although most packages are delivered by 6 p.m. Figure 3 shows the number of delivery attempts over the course of the simulated week. The delivery attempts increase towards the middle of the week at which they remain relatively stable. This can be attributed to two facts: Firstly, logiTopp currently does not account for additional delivery attempts resulting from the previous week. Secondly, orders placed during the weekend are not processed until the next business day, which also results in more deliveries towards the middle of the week.

One of the benefits of the integrated modelling approach is that the model can evaluate the agents' locations at all times during the simulation. This allows us to model different states of delivery on a microscopic level. Figure 4 shows the different states of delivery for one simulated day. Because agents are modelled in the context of their household, we can differentiate if the package was delivered to the agent who ordered the package or to a household member or parcel locker. In all cases, the deliveries are considered successful.

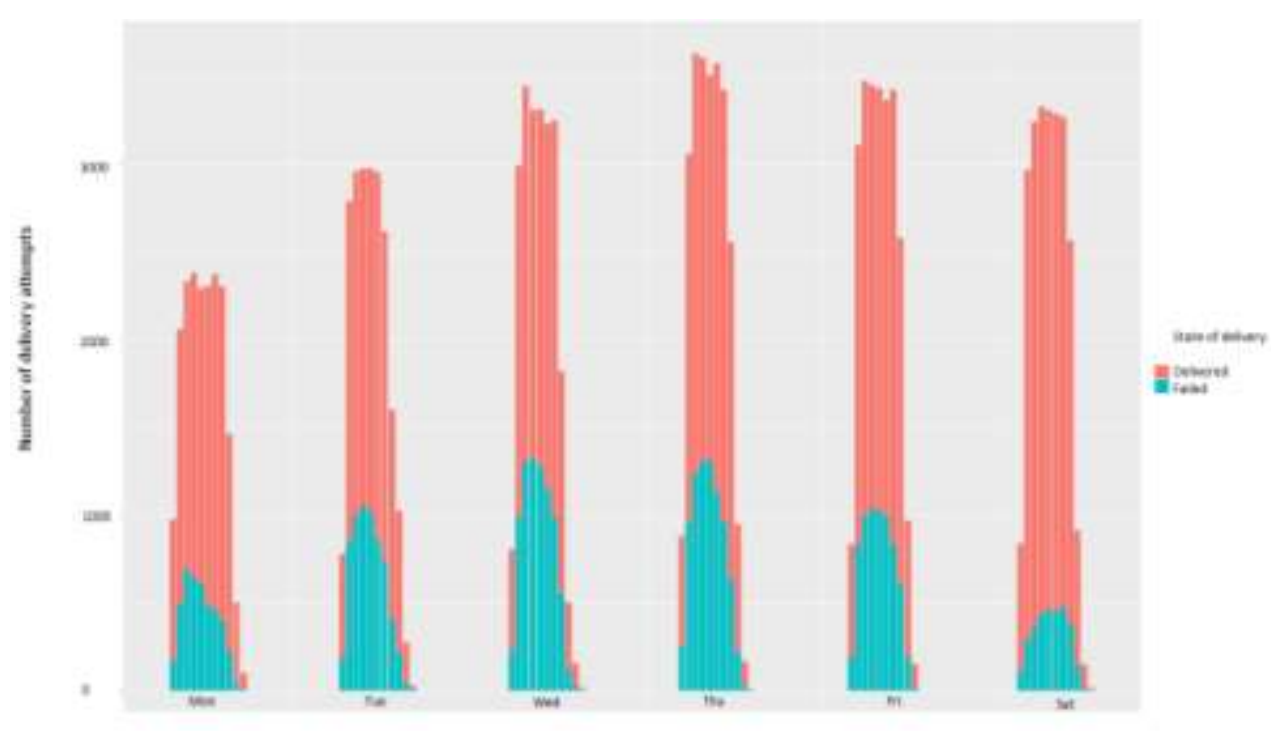

Figure 3 - Delivery attempts over the week 


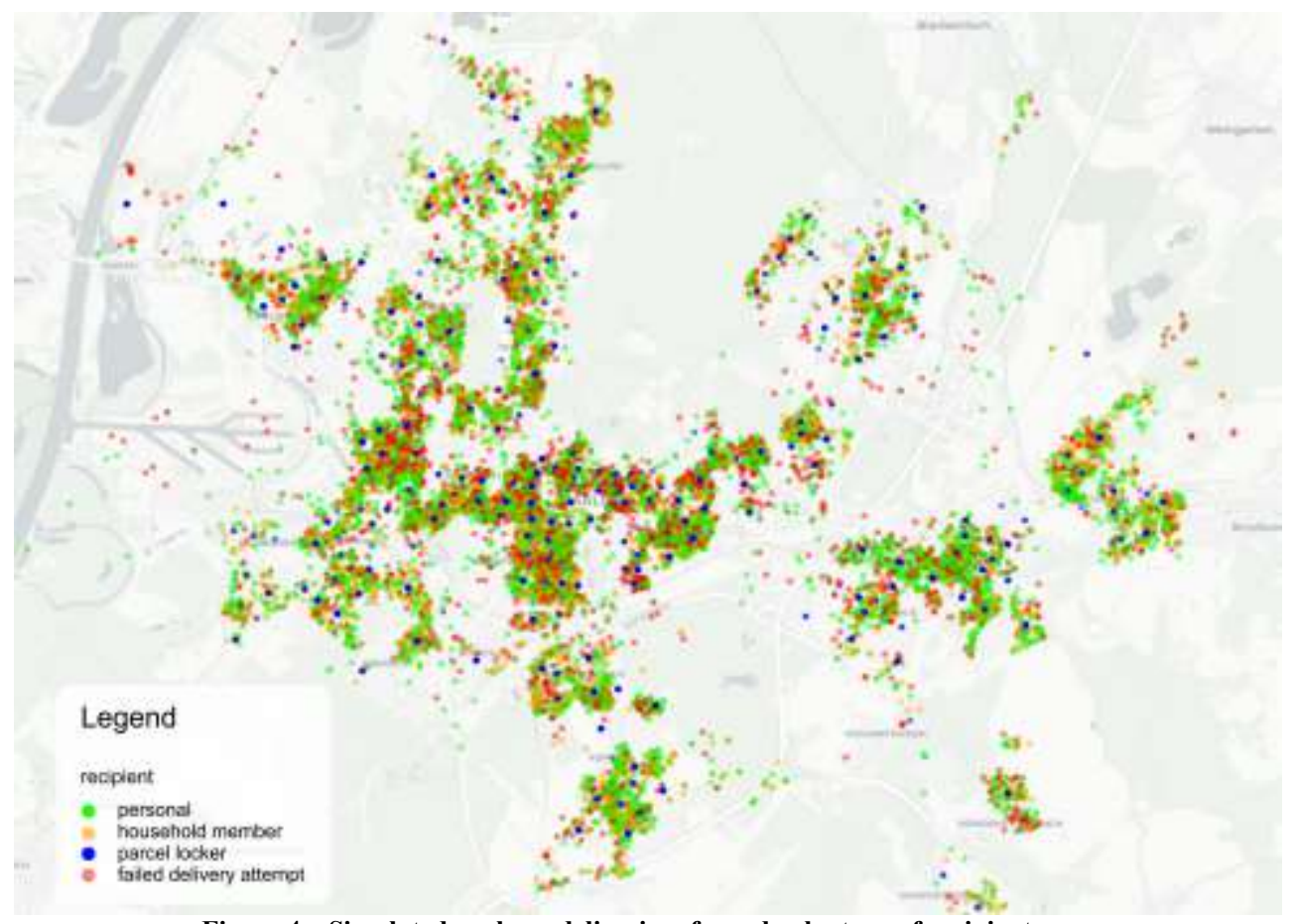

Figure 4 - Simulated package deliveries of one day by type of recipient

We have calculated the success rates of deliveries of the currently implemented model and visualised them both in terms of time and space. The success rate is defined as the number of successfully delivered packages over all deliveries. Figure 5 shows the delivery success rate for each hour of the day over the course of the simulation week. The plot shows that the success rates are lower in the morning and early afternoons of working days. This effect is reversed on Saturday due to receiving agents being at home in the morning able to receive packages and having more leisure activities planned later in the day and thus cannot receive packages then.

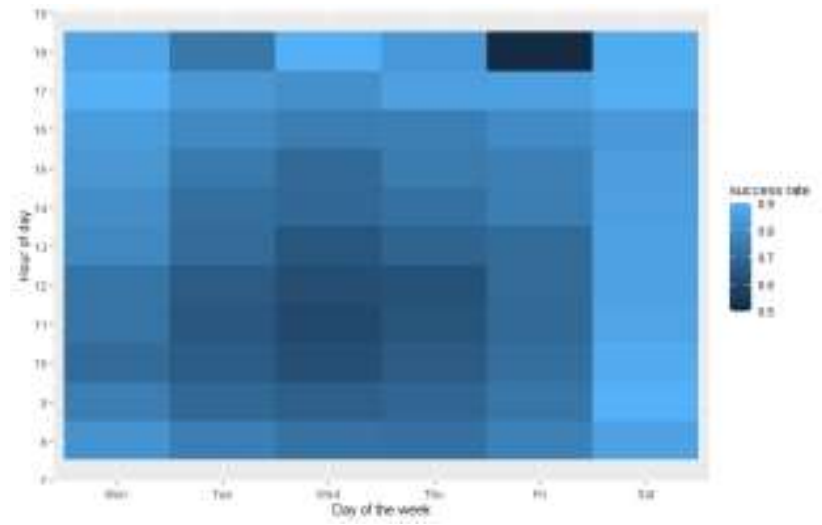

Figure 5 - Temporal distribution of the delivery success rate

Figure 6 shows the delivery success rate in each traffic analysis zone (TAZ) of the model. The plot allows for a spatial analysis of delivery success rates and could help policy makers and delivery service providers identify possible locations for e.g. parcel lockers which help mitigate problems of failed deliveries.

Figure 6 - Spatial distribution of the delivery success rate in TAZs of the model area
The simulated packages are delivered by 190 delivery agents and vehicles, respectively. The summary statistics of their tours is presented in Table 2 .

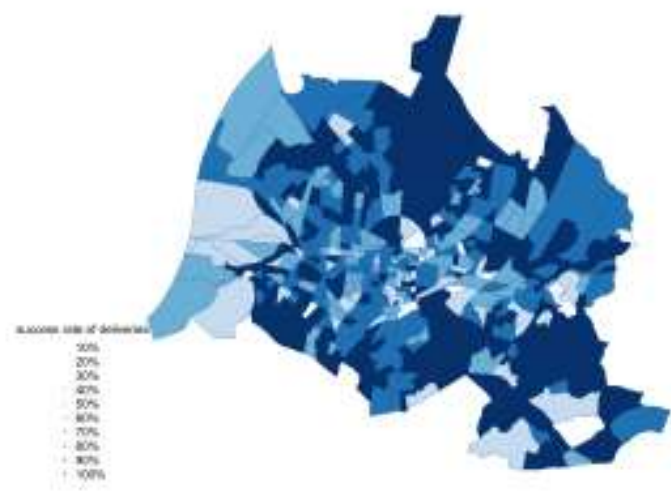

Table 2 - Summary statistics of delivery tours

\begin{tabular}{lllll}
\hline & \multicolumn{4}{c}{ variable } \\
\cline { 2 - 4 } & $\begin{array}{l}\text { \# of } \\
\text { stops }\end{array}$ & first stop & last stop & $\begin{array}{l}\text { tour } \\
\text { duration }\end{array}$ \\
\hline Min. & 4 & $08: 03: 00$ & $10: 06: 00$ & 53 min \\
$1^{\text {st }} \mathrm{Qu}$. & 148 & $08: 28: 00$ & $15: 22: 00$ & $6 \mathrm{~h} 34 \mathrm{~min}$ \\
Median & 160 & $08: 51: 30$ & $16: 00: 00$ & $7 \mathrm{~h} 9 \mathrm{~min}$ \\
Mean & 156.3 & $08: 50: 22$ & $16: 00: 14$ & $7 \mathrm{~h} 10 \mathrm{~min}$ \\
$3^{\text {rd }} \mathrm{Qu}$. & 173 & $09: 08: 45$ & $16: 39: 00$ & $7 \mathrm{~h} 49 \mathrm{~min}$ \\
Max. & 174 & $15: 36: 00$ & $19: 34: 00$ & $9 \mathrm{~h} \mathrm{57} \mathrm{min}$ \\
\hline
\end{tabular}

As the distribution of number of packages is an input variable for logiTopp, the results are not surprising and reflect realistic vehicle capacities. There are some outliers which we allow for due to larger shipments. However, the number of packages and therefore, shipment size are currently solely based on the input distribution as we have not yet implemented a shipment size model. 
Most of the delivery agents start their tours between 8 and 9 a.m. Three tours are started later than 10 a.m. which are all shorter tours with subsequently larger packages. The same holds true for the four tours that finish before noon. Most of the tours, however, are finished between 3 and 5 p.m. Considering the tour durations, we can see that logiTopp simulates the working day of the delivery agents realistically. Most tours are shorter than 8 hours which is realistic as delivery agents also spend some time of their working day loading and unloading at the beginning and end of the tours, respectively.

\section{Conclusion and Future Work}

This paper presents an integrated approach of modelling lastmile deliveries in an agent-based travel demand model. It is intended to account for reciprocal effects between online shopping behaviour and last-mile deliveries.

The framework allows for simultaneous simulation of private trips and last-mile deliveries and thus a realistic model of delivery conditions. Results show that the success rate of deliveries is temporally distributed as expected: it is low during working hours when no household members are at home to receive a package and high on Saturday when agents are more likely to receive a package. The spatial distribution of the success rates allows for an indication of where parcel lockers would be beneficial. However, these results are solely model based and would have to be checked against real world data. The model currently only includes one delivery strategy. As we include the real parcel distribution centres in the study area, we can consider individual delivery strategies which we will implement in a future version of logiTopp.

Currently, agents who chose to have packages delivered to their place of work have to be at work to receive the present. In reality, this is not always the case as front desks of large firms or colleagues at smaller companies will usually receive the package. We will include this in future models. Another delivery method currently not implemented is the use of designated delivery locations on the property of the agent who ordered the package. This method has become increasingly popular during the Covid-19 pandemic as it allows for contactless delivery. This method might mitigate a lot of failed deliveries as the receiving person does not need to be home to receive the package in person. These effects will also be scope of future analysis.

The work presented in this paper reflects the calibrated status quo model of urban package deliveries in Karlsruhe, Germany. The approach allows for detailed analysis of different scenarios. The scope of application includes both changes in delivery strategies and methods. Based on our model, we are able to consider and analyse findings from the literature regarding e.g. the optimal locations for parcel lockers [3]. The results allow for evaluation on the tour level meaning that possible emission reduction effects of different delivery strategies could be considered. One of such a strategy could be to integrate different locations as parcel delivery and pick-up points which is already common practice for many delivery services.

Furthermore, we are able to consider changes in (online) shopping behaviour and subsequent effects on travel patterns. The agent-based modelling framework allows for a wide array of scenario analyses. Future application of logiTopp will include the implementation of micro-hubs and delivery by cargo-bikes and their effect on the transportation system and $\mathrm{CO}_{2}$ emissions.

\section{Acknowledgments}

This paper stems from research within the project "Profillregion Mobilitätssysteme Karlsruhe" funded by the Ministry of Science, Research and the Arts BadenWürttemberg, the Ministry of Economic Affairs, Labour and Housing Baden-Württemberg and the Fraunhofer-Gesellschaft as a High-Performance center.

We would also like to thank the anonymous reviewer for providing helpful feedback and comments to improve this manuscript.

\section{References}

[1] K. Esser, J. Kurte, and K. KE-Consult Kurte \& Esser GbR, 'KEP-Studie 2020 - Analyse des Marktes in Deutschland', 2020 https://www.biek.de/files/biek/downloads/papiere/BIEK _KEP-Studie_2020.pdf

[2] M. Klumpp, 'Electric Mobility in Last Mile Distribution', in Efficiency and Innovation in Logistics, U. Clausen, M. Hompel, and J. F. Meier, Eds. Cham: Springer International Publishing, 2014, pp. 3-13. doi: 10.1007/978-3-319-01378-7_1.

[3] Y. Deutsch and B. Golany, 'A parcel locker network as a solution to the logistics last mile problem', International Journal of Production Research, vol. 56, no. 1-2, pp. 251-261, 2018, doi: 10.1080/00207543.2017.1395490.

[4] A. Conway, P.-E. Fatisson, P. Eickemeyer, J. Cheng, and D. Peters, 'Urban micro-consolidation and last mile goods delivery by freight-tricycle in manhattan: opportunities and challenges', Transportation Research Board 91st Annual Meeting, 2012.

[5] C. Fikar, P. Hirsch, and M. Gronalt, 'A decision support system to investigate dynamic last-mile distribution facilitating cargo-bikes', International Journal of Logistics Research and Applications, vol. 21, no. 3, pp. 300-317, 2018, doi: 10.1080/13675567.2017.1395830.

[6] M. Bok and L. Tavasszy, 'An empirical agent-based simulation system for urban goods transport (MASSGT)', Procedia Computer Science, vol. 130, pp. 126133, 2018, doi: 10.1016/j.procs.2018.04.021.

[7] M. Bok, L. Tavasszy, and S. Thoen, 'Application of an empirical multi-agent model for urban goods transport to analyze impacts of zero emission zones in The Netherlands', Transport Policy, 2020, doi: 10.1016/j.tranpol.2020.07.010.

[8] J. Joubert, P. J. Fourie, and K. Axhausen, 'A large-scale combined private car and commercial vehicle agentbased traffic simulation', Arbeitsberichte Verkehrs- und Raumplanung, vol. 560, 2009.

[9] S. Schröder and G. Liedtke, 'Towards an integrated multi-agent urban transport model of passenger and freight', Research in Transportation Economics, no. 64, pp. 3-12, 2017. 
[10] A. Alho, B. K. Bhavathrathan, M. Stinson, R. Gopalakrishnan, D.-T. Le, and M. Ben-Akiva, 'A multiscale agent-based modelling framework for urban freight distribution', Transportation Research Procedia, vol. 27, pp. 188-196, 2017, doi: 10.1016/j.trpro.2017.12.138.

[11] T. Sakai et al., 'SimMobility Freight: An agent-based urban freight simulator for evaluating logistics solutions', Transportation Research Part E: Logistics and Transportation Review, vol. 141, p. 102017, 2020, doi: 10.1016/j.tre.2020.102017.

[12] P. L. Mokhtarian, 'A conceptual analysis of the transportation impacts of B2C e-commerce', Transportation, vol. 31, pp. 257-284, 2004.

[13] L. Bönisch, S. Behren, B. Chlond, and P. Vortisch, 'Modification of Travel Behavior by E-Commerce? Capturing Relevant Factors by an Adapted Survey Approach Based on Previous Research', 99th Transportation Research Board Annual Meeting, Washington D.C., 2020.

[14] N. Mallig, M. Kagerbauer, and P. Vortisch, 'mobiTopp-a modular agent-based travel demand modelling framework', Procedia Computer Science, vol. 19, pp. 854-859, 2013.

[15] N. Mallig and P. Vortisch, 'Modeling travel demand over a period of one week: The mobiTopp model', arXiv preprint arXiv:1707.05050, 2017.

[16] T. Hilgert, M. Heilig, M. Kagerbauer, and P. Vortisch, 'Modeling week activity schedules for travel demand models', Transportation Research Record, vol. 2666, no. 1, pp. 69-77, 2017.

[17] KIT-IfV, logiTopp. 2021. [Online]. Available: https://github.com/kit-ifv

[18] J. E. Beasley, 'Route first - cluster second methods for vehicle routing', Omega, vol. 11, no. 4, pp. 403-408, 1983.

[19] D. Michail, J. Kinable, B. Naveh, and J. V. Sichi, 'JGraphT-A Java Library for Graph Data Structures and Algorithms', ACM Transactions on Mathematical Software (TOMS), vol. 46, no. 2, pp. 1-29, 2020.

[20] BIEK: Bundesverband Paket und Expresslogistik e.V., Ed., 'KEP-Studie 2020 - Analyse des Marktes in Deutschland', 2020. 